\title{
Emotional and General Intelligence: Characteristics, Meeting Points and Missing Links
}

\author{
Leonard I. Ugwu, Ph.D \\ Department of Psychology, University of Nigeria, Nsukka, Nigeria \\ E-mail: leonify@yahoo.com
}

Received: January 25, 2011 Accepted: February 11, 2011 doi:10.5539/ass.v7n7p137

\begin{abstract}
Emotional and general intelligence are two entangled knots begging for careful and successful separation by scientists and researchers in the field of Psychology. Although the two constructs overlap and perform similar functions in our lives, they can be separated in terms of their assessment, conceptualization and characteristics. Chief proponents of emotional intelligence believe that it makes people better students, better employees, better managers, better soldiers etc. The above assertion suggests that overall emotional intelligence contributes to individual success in life over and above the level attributable to general intelligence. Although the debates generated by the introduction of emotional intelligence in the field of psychology have not been laid to rest, the author contends that an individual with optimum level of the two constructs will be more successful and better adjusted to life.
\end{abstract}

Keywords: Emotional intelligence, General intelligence

\section{Introduction}

Emotional intelligence was coined by a Yale Psychologist Peter Salovey and John Mayer of University of New Hamphire fifteen years ago. They used the concept to describe qualities like understanding one's own feelings, empathy for the feelings of others and the individual's ability to regulate his or other people's emotion in a way that enhances living. Since the introduction of emotional intelligence in the field of psychology, it has generated a lot of debates from experts as to whether emotional intelligence is a component of general intelligence or an opposite of general intelligence. Epsteim and Meier (1989) disagreed that emotional intelligence is a component of general intelligent, pointing out that the two constructs are related and maintain lateral peership. Hein (2003) in his article titled "the dark side of emotional intelligence" has argued that emotional intelligence is a component of general intelligence, pointing out that emotional intelligence (EI) is what Cantor and Kilhstrom (1987) first called social intelligence, which is a component of the traditional general intelligence. Many writers in area of intelligence made effort to separate EI from general intelligence but this is not by any means easy since the two concepts are characterized by great conceptual diversity. The definitional problems stem from the fact that both general and emotional intelligence are influenced by culture. However, its conceptual diversity not withstanding, Salovey and Mayor (1990) defined EI as "the ability to monitor one's own and others feelings and emotions, to discriminate among them and to use this information to guide one's thinking and actions. Goleman (1995) described emotional intelligence as a mix of skills, such as awareness of emotions; traits, such as persistence and zeal; and good behaviour. He summarized the collection of intelligence (E1) as the best predictor of success in life, redefining it as being "smart". This definition suggests that individual's success in life is accounted more by one's ability to process, understand, manage, and use emotions in other to benefit from it. The implication of the above definition by Gibb is that when it comes to predicting people's success in adapting to life, brainpower as measured by $1 \mathrm{Q}$ and standardized achievement tests may actually matter less than the qualities of mind once thought of as "character". For Goleman (1997):

"Emotional intelligence gives one a competitive edge ...Having great intellectual abilities may make one a superb fiscal analyst or legal scholar, but highly developed emotional intelligence will make you a candidate for Chief Executive (CEO) or a brilliant trial Lawyer: (p. 1).

Thilam and Kirby (2002) investigated the impact of emotional and general intelligence on individual performance and concluded that overall emotional intelligence (emotional perception and emotional regulation) uniquely explained individual cognitive-based performance over and beyond the level attributable to general intelligence. With increasing usage of emotional intelligence by managers and scholars, Mayer and Salovey 
(1997) recognized their earlier definition as inadequate and stated that emotional intelligence reflects not a single trait or ability but, rather, a composite of distinct emotional reasoning abilities: perceiving, understanding, and regulating emotions. Cobb and Mayer (2003) characterized emotional intelligence as "powerful", at times "more powerful", and even "twice as powerful" as 1Q; it de-emphasized the importance of general 1Q and promised to level the playing field for those whose cognitive abilities might be wanting. After an extensive review of literature on emotional intelligence, Cobb and Mayer (2003) summarized it thus:

"Among the ingredients for success, 1Q counts for about $20 \%$; the rest depends on everything from environment to luck to the neural pathways that have developed in the brain over millions of years of human evolution" (p.3).

\section{Characteristics of Emotional and General Intelligence}

The concept of emotional intelligence, just like general intelligence, does not have one generally accepted definition. This is because it all depends on what a given culture considers as being indices of emotional intelligence since emotional intelligence is a measure of an understanding of responsible behaviour at a given social context. Each cultural group has a set of behaviours indicative of emotional intelligence, which points to the fact that people from different cultures may perceive emotional expressions differently. The implication is that any assessment technique or procedure that is to be used in assessing people's level of emotional intelligence should assess the abilities recognized by the culture of the client as constituting qualities of emotional intelligent behaviour. What are regarded as positive or negative emotions, for instance, differ from culture to culture. Eyo (2003) captures those differences vividly when he described the different emotional expressions across cultures. He stated that cultural conventions have some influences on emotional expressions, such as smiling, laughing, weeping, frowning, expressions of anger and fear, etc. all of which are forms of behaviour. To buttress his point, he described how Malinese chieftains are said to frown very fiercely when greeting each other at festival occasions which is typical of their culture, whereas Nigerians are known to be highly exuberant and demonstrative - laughing loudly and shaking hands or embracing each other with a great deal of intensity.

Emotional and general intelligence are psychological constructs that lend themselves to different interpretations by people interested in them. With so many diverse definitions of intelligence and cultural influence on the interpretation of emotional intelligent behaviour, it is perhaps not surprising that we cannot agree on how to measure and interpret emotional intelligence. This is because people with differing cultures and norms tend to socialize and maintain different emotions and individual's emotional intelligence is evaluated on the basis of acceptable mode of expressions of emotion in that culture. The implication of this is that without intimate knowledge of the norms and culture of a particular society one will not be able to find the logic in its systems of emotional expression. Therefore, in the World over, it will be difficult to obtain a generally accepted mode of intelligent emotional expression.

Another characteristic of emotional intelligence worthy of explanation is its components. Mayer and Cobb (2000) identified four components of emotional intelligence, which include perception, understanding, and facilitation of thought and management of emotions. Perceiving emotions consists of recognizing and interpreting the meaning of various emotional states, as well as their reactions to other sensory experiences. Understanding emotions involves comprehension of how basic emotions are blended to form complex emotions, and how emotions are affected by events surrounding one's emotional experiences. Facilitation of thought refers to one's ability to capture emotions and give meanings to them as soon as they are expressed. Management of emotions refers to one's ability to control emotions in one's own and in others. These components of emotions suggest that emotional intelligence reflects not a single trait or ability but, rather, a composite of distinct emotional reasoning abilities (Thilam \& Kirby, 2002). The implication of this is that emotional intelligence is a form of intelligence, not a component of general intelligence that involves the ability to monitor one's own and other's feelings and emotions, to discriminate among them and to use this information to guide one's thinking and actions.

Furthermore, emotional reactions can easily be detected through outward expressions and facial look and as well possesses the characteristics of being contagious. In his own words, FridLund (1994) described the face as a switch on a railroad tracks. It affects the trajectory of the social interaction the way the switch would reflect the path of the train". This suggests that there is a link between facial expression and emotion, but it is not a one-to-one kind of relationship. For instance, there are many situations where emotions are expressed, yet no corresponding facial expression is displayed; and there are times when a facial expression appears with no corresponding emotion. This is referred to as surface emotion. But in contrast, 1Q expresses itself only when presented with intelligence tests and adaptation problems. Emotion is contagious because emotional reactions 
tend to elicit similar expression of emotions from others. So, a smile may beget smile from others just as yawning from one person elicits yawning from others. But this is not the case with general intelligence; general intelligence is an inherited traits and not contagious.

\section{Meeting Points}

When we define emotional intelligence as one's ability to perceive, understand and manage one's emotions and emotions of others, we have tacitly acknowledged that 1Q is a critical factor in emotional expression. For instance, ability is a component of general intelligence, which suggests that humans posses the ability traits that are relevant to controlling emotions. Having the will power to control emotion is a function of general intelligence, particularly in a situation where an expression of the desirable emotional reaction is sine qua non to the attainment of the valued goal. Therefore, the bedrock of emotional intelligence is one's ability to delay gratification or regulate one's emotions in order to attain a desired goal.

Secondly, emotional intelligence (EI) looks and behaves like other intelligences, each as verbal intelligence, which develops with age. The effective development of verbal intelligence can be facilitated or hindered by the environment. A newborn baby has no known language; he babbles and gradually masters the language spoken by his parents and people around him. The same is applicable to emotion, which is affected by age and environment. Although a child at birth is equipped with emotions, he does not know the right emotion to express or how to regulate emotion for healthy living. Parents and significant others provide such skills. The point being made here is that EI and verbal intelligence can be developed even when they have their root in inheritance.

Another meeting point of EI and QI is manifest when we are emotionally tensed up. For instance, emotional experience alters thought processes by beclouding them. That explains why an individual who is tensed up performs poorly in intellectual matters. Under emotion, speech may be impaired as the person may make more grammatical errors, sometimes stammers, and veraciously use non-verbal cues to convey his feelings. The big question begging for an answer is: Does emotion govern intelligence and vice versa? It is the belief of the writer that the relationship is bi-directional: people high in intelligence can suppress irrational manifestation of emotion in one circumstance, while emotion in another circumstance can becloud reasoning. If emotional intelligence can mask general intelligence and vice versa, under different circumstance, then they must have meeting points.

In search of the meeting points, we have to recall that emotional life grows out of the area of the brain called the Limbic system, specifically the amygdale, whence come delight and disgust. Lust grows from the limbic system; love from the neocortex. Animals like reptiles that have no neocortex cannot experience anything like maternal love (LeDoux, 1996); this explains why baby snakes have to hide to avoid being eaten by their parents. But humans with their well-developed neocortex show affection and love to the younger ones. The more connections between limbic system and the neocortex, the more emotional responses are possible. According to LeDoux (1996), the short circuit in the brain allows emotions to initiate action before the intellect gets a chance to intervene. This suggests that at one extreme of the same continuum is the limbic system while at the other end is the cerebral hemisphere with the connecting pathways between them. Depending on the origin, the nerve impulses then travel along these nerve endings to convey messages from one source to the other and vice-versa.

Furthermore, emotional intelligence can be measured in terms of individual's ability to delay gratification. Therefore, endurance is the corner stone of emotional intelligence. It is a sign, in short, of emotional intelligence and such does not show up on an IQ test even when we know it to be a factor in general intelligence. In fact, a search for their meeting points is still a mirage, as both emotional and general intelligence appear to have invisible meeting points. In sum, emotional intelligence, originating from the limbic system and general intelligence, which originates from the cerebral cortex, are like two rivers with a connection channel, each river being inhabited by a hippopotamus. When one of the rivers is stirred up by the hippopotamus, the torrent passes through the channel and submerges the other river and vice-versa.

\section{Missing Links}

There are unsettled questions till date as to whether emotional and general intelligence are interrelated or distinct concepts with no relationship. Some of these questions begging for answers in the field of emotional intelligence are: why is it that the smartest kid in the class will probably not end up the most successful in life? Why do some people wear smiling faces even in the face of troubles, while others would flay up under the same condition? Do emotions affect general intelligent behaviour and vice-versa? Is relationship between EI and general intelligence causal or associative? Why are emotionally intelligent people more effective in achieving better marriages, in successfully raising their children better, and in achieving better mental and physical well-being than their counterparts who have more IQ but low Emotional Quotient (EQ)? Can emotional intelligence be assessed the same way as general intelligence? These are knotty questions which researchers interested in this field of study 
have not been able to provide answers to satisfactorily, suggesting that there may be some ingredient missing. This is in support of Igbo adage, which says, "Passing six is not passing sense," that is, attainment of greater height in academics does not depict wisdom. If a high degree of emotional intelligence is a prerequisite for outstanding achievement there ought to be no better place to find it than in leadership. But Hein (2005) reported that not every man who reached the pinnacle of American leadership was a gleaming example of self-awareness, empathy, impulse control and all the other qualities that mark an elevated EQ. Holmes described Roosevelt as "a second-class intelligent, but first-class "temperament" but described Thomas Jefferson as the "most perfect blend of intellect and warmth of personality. Richard Ellis, a professor of politics at Oregon University says, "Bill Clinton is very brainy but his emotional intelligence is shaky... as for controlling his impulse, Clinton is terrible". "As for Richard Nixon, he was the "worst cases" on any EQ scale of presidents". These were American Presidents in the past with differing IQ and EQ but successfully transformed America. The lesson we have learnt here is that a good measure of IQ and EQ are needed for outstanding achievement. The issue of emotionally intelligent people achieving better marriages and successfully raising their children more than generally intelligent person is obvious. Marriage as well as raising children involves social relationship and individuals should be emotional intelligent to the extent that they have the ability to control their emotions.

In sum, there are some areas where EI and IQ meet, but the meeting point is not clear-cut. On the contrary, there are areas of missing links - some individuals are generally intelligent but low in EI and vice-versa. How do we explain these? An individual with both optimum IQ and EQ will make a "better" success in Life.

\section{References}

Cantor, N and Kihlstrom, J. F. (1987). Personality and Social Intelligence. Rnglewood Cliff, NJ: Prentice-Hall.

Cobb, C. D. and Mayer, J. D. (2003). Emotional Intelligence: What the research says. In K. G. Duffy (ed.), Personal Growth and Behaviour. United States: McGraw-Hill.

Epsten, S. and Meier, P. (1989). Constructive thinking: A broad coping variable with specific components. Journal of Experimental Social Psychology, 28, 339-359.

Eyo, I. E. (2003). Determinants of Behaviour: Giving Meaning to the Psychological Concern with Behaviour. Nsukka: AP Publishers.

Fridlung, A. (1994). Human Facial Expression: An evolutionary View. San Diego CA Academic Press.

Gibbs, N. (1995, October 2). The EQ factor. Time, 146, 60-68.

Goleman, D. (1995). Emotional Intelligence: why it can matter more than IQ. New York: Bantam Books.

Hein, S. (2003). The Dark Side of Emotional Intelligence. Englewood Cliff, N.J: Prentice Hall.

Hein, S. (2005). History and Definition of Emotional Intelligence. Englewood Cliff, NJ: Prentice - Hall.

LeDoux, J. (1996). The emotional brain: The Mysterious Underpinnings of emotional life. New York: Touchstone.

Mayer, J. D. and Cobb, C. D. (2000). Educational Policy on emotional intelligence: Does it make sense? Educational Psychology Review, 12, 163- 183.

Mayer, J. D. and Salovey, P. (1997). What is emotional intelligence? In P. Salovey and D.J. Sluyter (eds.). Emotional development and emotional intelligence (Pp. 3 - 31). New York: Basic Books.

Salovey, P. and Mayer, J. D. (1990). Emotional intelligence. Imagination, Cognition, and Personality, 185 -211.

Thilam, L. and Kirby, S. (2002). Is Emotional intelligence an Advantage? An exploration of the impact of emotional and general intelligence on individual performance. The Journal of Social Psychology, 142(1), 133 143. 